NOTAS TECNICAS

\title{
Antecedentes del Sirex noctilio (Hymenoptera-Siricidae) en el Valle de Calamuchita, Cordoba, Argentina
}

\author{
Preliminary studies on Sirex noctilio (Hymenoptera-Siricidae) in pine plantations \\ in the Calamuchita Valley, Córdoba, Argentina
}

ADLIH LOPEZ, MARCELA DEMAESTRI, ESTEBAN ZUPAN, OMAR BAROTTO

Facultad de Agronomía y Veterinaria, Universidad Nacional de Río Cuarto, Argentina. E-mail: alopez@ayv.unrc.edu.ar

\begin{abstract}
SUMMARY
In 1994, the wood wasp parasite Sirex noctilio was identified in Pinus spp. growing in the Calamuchita Valley of Cordoba, Argentina. This insect causes direct and indirect damage to trees, eventually causing death. The larval activity consisting of feeding and injecting a phytotoxic mucus, coincides with infection of the symbiotic pathogenic fungus Amilostereum areolatum. The "pine borer wasp" has a commercial impact, since the export of infested pinewood is prohibited by international agreement, thus affecting the regional economy based on pine forest products. A research project at the Universidad Nacional de Rio Cuarto dealing with the prevalence, distribution and incidence of Sirex in the region was begun in 1996. We are also studying the bioecology and natural enemies of the insect, as well as the convenience of introducing a parasite nematode, Deladenus sincidicola, to try to managing the problem. The "pine borer wasp" was declared an agricultural pest for the Province of Cordoba in 1997.
\end{abstract}

Key words: Sirex noctilio, management, economic impact.

\section{RESUMEN}

En 1994 fue identificada Sirex noctilio F. (Hymenoptera-Siricidae) en las plantaciones de Pinus spp. (31.125 ha de Pinus elliottii, 3.738 ha de $P$. radiata y $P$. taeda) en el Valle de Calamuchita. La importancia de esta plaga radica en su acción directa por la actividad larval y los daños que indirectamente ocasiona por la inyección de un mucus fitotóxico y de esporas del hongo simbionte patogénico Amylostereum areolatum, lo que provoca que el árbol se seque. Además por tratarse de una plaga cuarentenana para los países que importan productos de nuestros bosques, su presencia restringirá la comercialización forestal con gran impacto en la economía local y regional. A partir del año 1996 en la Universidad Nacional de Rio Cuarto (UNRC) se comienza a investigar acerca de la presencia, distribución y grado de ataque en la región, su bioecología, controladores naturales e introducción del nemátodo Deladenus siricidicola, con el objetivo de establecer pautas de manejo de la "avispa barrenadora de los pinos". En 1997 se la declara "Plaga de la Agricultura" en la Provincia.

Palabras claves: Sirex noctilio, manejo-impacto economico.

\section{INTRODUCCION}

El Valle de Calamuchita, provincia de Córdoba, según el Mapa de Aptitud Forestal (Izurieta et al. 1993) posee 36.202 ha de pinos, las que representan el $10 \%$ de las plantaciones de coniferas del país, estimándose la superficie potencialmente apta para la forestación en 250.000 a 300.000 ha Se- gún la Dirección de Recursos Naturales Renovables (D.R.N.R), hacia 1982, de la superficie implantada 3.738 ha correspondían a Pinus radiata y 31.125 ha a Pinus elliottii y $P$. taeda (Izurieta et al. 1993).

En esta región, desde hace algunos años, según informaciones proporcionadas por los productores a un diario local, se comenzaron a obser- 
var los primeros árboles muertos con orificios en los troncos.

En febrero de 1994, técnicos de la D.R.N.R de la provincia de Córdoba capturaron un ejemplar de avispa, el que se presuponía como la causa de las mencionadas manifestaciones. Este insecto fue enviado a la Facultad de Ciencias Forestales de El Dorado (Misiones) e identificado como Sirex noctilio F. (Hymenoptera-Siricidae), "Avispa barrenadora del pino", siendo éste el primer registro en el Valle de Calamuchita.

La presencia de la avispa barrenadora en dichas áreas, donde la forestación es un importante recurso económico y ecológico, constituye un grave problema fitosanitario, y además una amenaza latente para las áreas no afectadas o de bajo nivel de ataque.

Es de destacar que la importancia de esta plaga radica en su acción directa y los daños que indirectamente ocasiona en acción conjunta con la actividad larval y por la inyección de un mucus fitotóxico y de esporas del hongo simbionte patogénico Amylostereum areolatum (que realiza la hembra al oviponer) que es fuente de alimentación para la larva de Sirex, lo que provoca que el árbol se seque y penetren hongos xilófagos secundarios que tornan a la madera no apta para el mercado, comenzando por los árboles más debilitados y siguiendo luego, cuando la presencia de este insecto es mayor, con los árboles más vigorosos.

En las plantaciones bien manejadas los daños no son graves, debido a que está comprobado que las forestaciones más afectadas son aquellas cuyas edades superan los 15 años, donde existe una alta densidad de plantas y donde se encuentran árboles debilitados por la alta competencia y falta de manejo (podas y raleos), estresadas por factores ambientales (sequías), incendios, etc. En el sur de Valle, sobre unas 11.000 ha forestadas, las mayores superficies implantadas se lograron entre los años 1968-1980 (entre 19 y 31 años de edad), de las cuales sólo 3.000 ha han sido manejadas con prácticas de poda y ocasionalmente raleo.

La curva de crecimiento de Pinus spp. tiene mayor incremento a partir de los doce años, esperando un retorno a los 20-25 años, cuando la madera posee la mayor calidad. Ante la aparición de la avispa barrenadora se debe realizar un raleo sanitario, lo que implica eliminar árboles que podrían tener un alto valor maderable y que no podrán llegar a la corta final. Este material raleado además debe extraerse del monte, chipearse, que- marse o secarse en estufa, para evitar la dispersión de la avispa, aumentando los costos de producción.

Por otra parte, por tratarse de una plaga cuarentenaria para los países que importan productos de nuestros bosques, su presencia restringirá aún más la comercialización forestal con gran impacto en la economía local y regional.

La dispersión de la avispa es inevitable, sobre todo en zonas como la nuestra, con especies altamente susceptibles, pudiendo introducirse además a lugares distantes con el transporte de madera por rutas donde no se cumplen las medidas cuarentenarias. Debido al alto potencial de daño de esta plaga, es importante encontrar medidas eficaces a los fines de poder manejar y retardar su dispersión, preservando la sanidad de nuestras forestaciones.

El uso de plaguicidas, particularmente los insecticidas, es considerado como uno de los factores perturbadores (índice de insostenibilidad) de mayor importancia en las zonas forestales, por lo cual se establece que la aplicación de medidas preventivas como manejo de controladores naturales e introducción de parasitoides, integradas con prácticas del monte dirigidas a mejorar la calidad de los árboles, son los componentes esenciales para enfrentar a la plaga.

\section{LEGISLACION VIGENTE EN LA PROVINCIA}

- Ley Provincial de Sanidad Vegetal 4987 (1968), propone la nomenclatura de agentes perjudiciales en la producción agrícola con su Decreto $\mathrm{N}^{\circ} 6373$ reglamentario de la Ley $\mathrm{N}^{\circ} 4967$.

- Decreto 675/96 de "Declaración de estado de Emergencia Sanitaria", el cual autoriza a los productores a realizar raleos sanitarios como medida preventiva sin la presentación previa de los requisitos exigidos en la legislación vigente, sin embargo no especifica cuáles son los medios con los que el productor deba eliminar el material afectado, sólo establece que sean los más convenientes y causen menor impacto ambiental.

- Resolución Ministerial $N^{\circ} 150 / 97$ de creación de la "Comisión de Prevención y Lucha Contra la Avispa Barrenadora de los Pinos" (Resol. $\mathrm{N}^{\circ} 150 / 97$ de Ministerio de Producción y Trabajo del Gobierno de la Pcia. de Córdoba), integrada por Secretaría de Agricultura, Ganadería y Recursos Renovables, la Universidad Na- 
cional de Córdoba, Universidad Nacional de Río Cuarto, Universidad Católica de Córdoba, INTA y representantes privados del área forestal.

- Decreto 1944/97 de Declaración de "Plaga de la Agricultura" a la "Avispa Barrenadora de los Pinos" (Sirex noctilio) en todo el territorio de la provincia de Córdoba de conformidad con la ley 4027 y su Decreto reglamentario 6373/79. El mismo propicia coordinar las medidas que tiendan hacia el manejo integrado de la plaga, a fines de prevenir su dispersión en las plantaciones y lograr el control de los focos existentes. Además propicia el estudio de la bioecología de Sirex en las condiciones agroecológicas de la región, promoviendo la investigación para detectar el control natural dentro de la entornofauna local.

\section{ACCIONES REALIZADAS}

La Universidad Nacional de Río Cuarto (UNRC), Facultad de Agronomía y Veterinaria, comenzó en 1996 a trabajar en el tema a pedido de un grupo de forestadores, representantes de la región, quienes solicitaron apoyo ante este problema.

Investigadores del Area Forestal elaboraron proyectos a desarrollar en la zona de influencia de la Universidad (Centro-Sur del Valle de Calamuchita), sobre el comportamiento de la plaga, su distribución y grados de ataque, presencia de controladores naturales, e introducción del nemátodo Deladenus siricidicola, entre otros, los cuales fueron aprobados y apoyados por la Secretaría de Ciencia y Técnica UNRC y del SAGyRR de la provincia de Córdoba en 1997 (López et al. 1997a, López et al. 1997b).

En junio de 1997 se realizó en Villa General Belgrano, Córdoba, la Reunión Interprovincial de la Avispa Barrenadora de los Pinos, con la participación de las provincias afectadas por Sirex, donde se conformó la Comisión Nacional de Lucha, con participación de representantes de las Direcciones de Recursos Naturales Provinciales, INTA (Instituto Nacional de Tecnología Agropecuaria), Universidades y sector privado con el fin de encarar un plan de acción conjunto en las áreas de investigación, capacitación, extensión, fiscalización y legislación a nivel nacional.

En marzo de 1998 se continúan las investigaciones y se realizó la presentación por parte de la UNRC del Plan de Lucha para la provincia de Córdoba ante las autoridades provinciales y los productores forestales. En agosto de 1999 se reitera ante la Agencia Córdoba Ambiente Sociedad del Estado (figura 1). (López et al. 1999, Demaestri et al. 1999, Zupan et al. 1999 a y b, López et al. 2000).

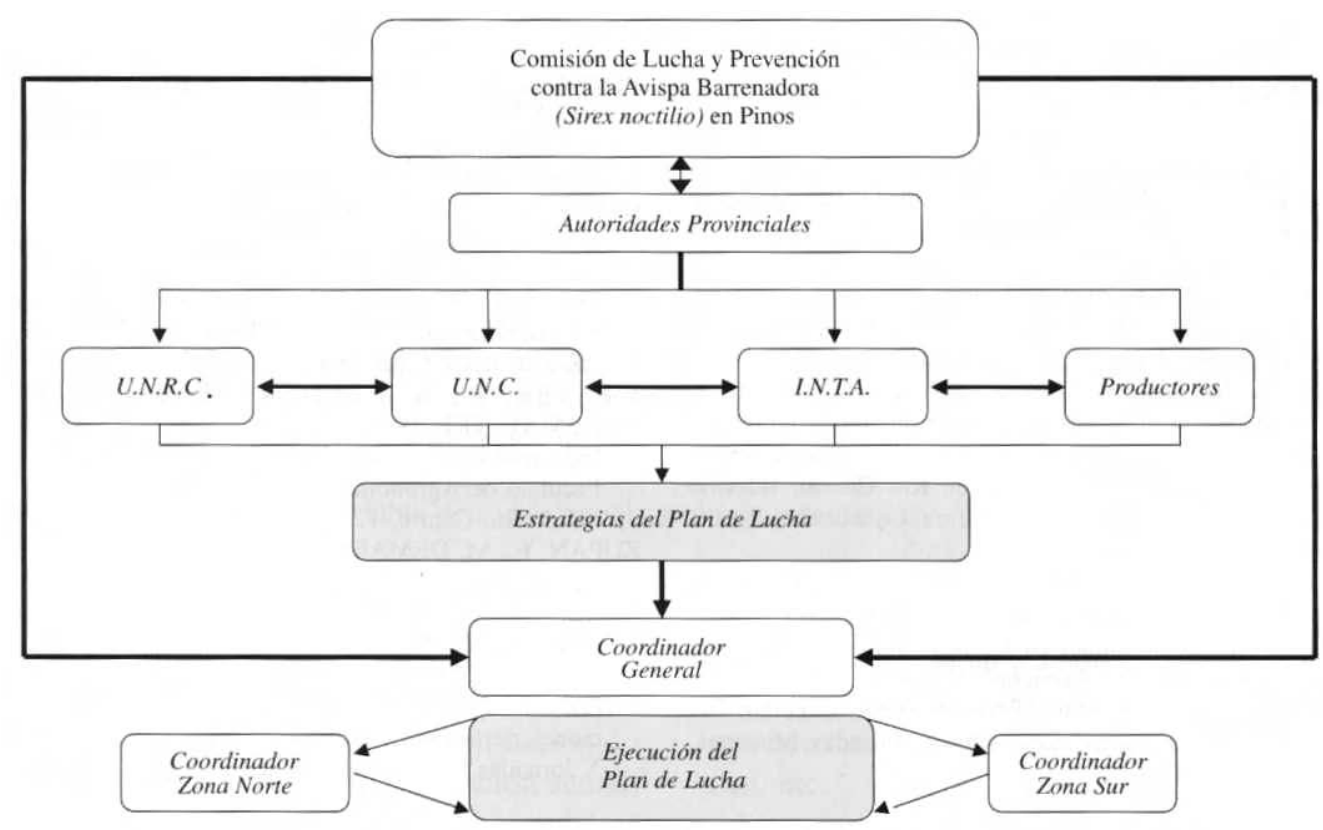

Figura 1. Propuesta de organigrama, plan de lucha Sirex noctilio.

Flowchart proposal, program of Sirex noctilio control. 
A principios de 1999 se aprobó un Proyecto de Investigación Aplicada (PIA), dentro del Proyecto Forestal de Desarrollo -SAGPyA con el fin de ampliar el trabajo de la UNRC a toda la región del Valle de Calamuchita.
La investigación, la difusión de técnicas de prevención, el control localizado de las infecciones y el manejo de las plantaciones forestales, conducirán a reducir las poblaciones actuales de Sirex noctilio a niveles económicamente aceptables y poder convivir con su presencia (figura 2).

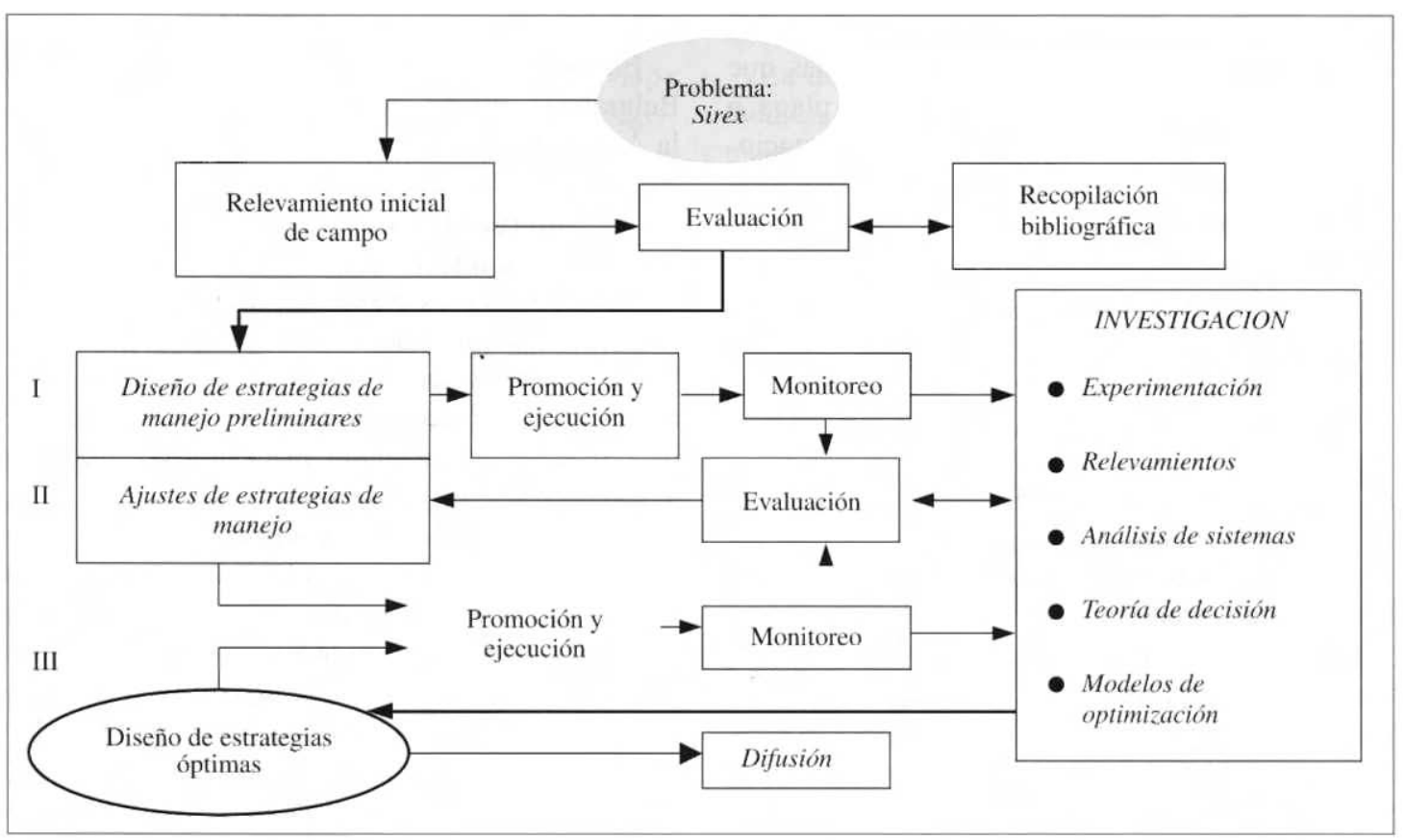

Figura 2. Estrategias de intervención en Sirex noctilio. Intervention strategy for Sirex noctilio.

\section{BIBLIOGRAFIA}

DEMAESTRI, M., E. ZUPAN, A. LOPEZ. 1999. Experiencias de manejo de Sirex noctilio F. en el Sur del Departamento Calamuchita. Córdoba- X Jornadas Fitosanitarias Argentinas, pp. 281.

IZURIETA, G., D. ABUD, J. IZAURALDE. 1993. Plantaciones de Pinos de la Provincia de Córdoba. Congreso Forestal Argentino y Latinoamericano Paraná, 7 p.

LOPEZ, A., M. DEMAESTRI, E. ZUPAN, G. BALBI, J. GONZALEZ. 1997a. Presencia, Distribución y Grado de Daño del Sirex noctilio (F) en el Valle de CalamuchitaCórdoba. Universidad Nacional de Río Cuarto. Informe presentado a Secretaría de Agricultura, Ganadería y Recursos Renovables. Córdoba, 11 p.

LOPEZ, A., M. DEMAESTRI, E. ZUPAN, L. MUGAS. 1997b. "Distribución y grado de daño producido por Sirex noctilio (Hymenoptera- Siricidae) en las forestaciones del sur del Valle de Calamuchita- Córdoba". II Congreso Forestal Argentino y Latinoamericano. "Forestar y Crecer" Tomo: Comisión Bosques Cultivados- A.Fo.A. Posadas Misiones. ISSN: 0329-1103.
LOPEZ, A., M. DEMAESTRI, E. ZUPAN, O. BAROTTO, E. TRUMPER, R. BALBOA. 1999. "Sirex noctilio en el Valle de Calamuchita-Córdoba". X Jornadas Fitosanitarias Argentinas, pp. 280.

LOPEZ, A., M. DEMAESTRI, E. ZUPAN, O. BAROTTO, G. BALBI. 2000. Application of a Geographical Information System to the Study of Sirex noctilio F. (HymenopteraSiricidae) in Calamuchita Valley, Córdoba, Argentina. The XXI International Congress of Entomology - Fox de Iguazu - Brasil. Book I, pp. 489.

WEAVER, P., B. CHEDZOY, P. INGARAMO, M. DEMAESTRI. 1994. Situación de la Actividad ForestoIndustrial en el Valle de Calamuchita. Informe Técnico. Facultad de Agronomía y Veterinaria, Universidad Nacional de Río Cuarto, $12 \mathrm{p}$.

ZUPAN, E., M. DEMAESTRI, A. LOPEZ. 1999a. "Relación entre emergencia de adultos del parasitoides Ibalia leucospoides H. y Sirex noctilio F. en el Valle de Calamuchita-Córdoba. X Jornadas Fitosanitarias Argentinas, Jujuy, pp. 279.

ZUPAN, E., M. DEMAESTRI, A. LOPEZ. 1999b. Observaciones de trozas de árboles atacados por Sirex noctilio $\mathrm{F}$. X Jornadas Fitosanitarias Argentinas, pp. 278. 